TRANSACTIONS OF THE

AMERICAN MATHEMATICAL SOCIETY

Volume 348, Number 8, August 1996

\title{
ON QUADRATIC FORMS OF HEIGHT TWO AND A THEOREM OF WADSWORTH
}

\author{
DETLEV W. HOFFMANN
}

\begin{abstract}
Let $\varphi$ and $\psi$ be anisotropic quadratic forms over a field $F$ of characteristic not 2. Their function fields $F(\varphi)$ and $F(\psi)$ are said to be equivalent (over $F$ ) if $\varphi \otimes F(\psi)$ and $\psi \otimes F(\varphi)$ are isotropic. We consider the case where $\operatorname{dim} \varphi=2^{n}$ and $\varphi$ is divisible by an $(n-2)$-fold Pfister form. We determine those forms $\psi$ for which $\varphi$ becomes isotropic over $F(\psi)$ if $n \leq 3$, and provide partial results for $n \geq 4$. These results imply that if $F(\varphi)$ and $F(\psi)$ are equivalent and $\operatorname{dim} \varphi=\operatorname{dim} \psi$, then $\varphi$ is similar to $\psi$ over $F$. This together with already known results yields that if $\varphi$ is of height 2 and degree 1 or 2 , and if $\operatorname{dim} \varphi=\operatorname{dim} \psi$, then $F(\varphi)$ and $F(\psi)$ are equivalent iff $F(\varphi)$ and $F(\psi)$ are isomorphic over $F$.
\end{abstract}

\section{INTRODUCTION}

Let $F$ be a field of characteristic different from 2. By the function field of a (non-degenerate) quadratic form $\varphi$ over $F$ we mean the function field of the projective variety $\varphi=0$ if $\operatorname{dim} \varphi \geq 2$ and $\varphi$ not isometric to the hyperbolic plane $\mathbb{H} \simeq\langle 1,-1\rangle$. We denote this function field by $F(\varphi)$ and put $F(\varphi)=F$ if $\varphi \simeq \mathbb{H}$ or $\operatorname{dim} \varphi=1$. $F(\varphi) / F$ has transcendence degree $\operatorname{dim} \varphi-2$, and the extension is purely transcendental iff $\varphi$ is isotropic. We call two field extensions $L / F$ and $K / F$ equivalent over $F$ if there exist $F$-places $L \rightarrow K \cup\{\infty\}$ and $K \rightarrow L \cup\{\infty\}$. M. Knebusch showed in [16] that for two quadratic forms $\varphi$ and $\psi$ we have that $F(\varphi)$ is equivalent to $F(\psi)$ iff $\varphi$ is isotropic over $F(\psi)$ and $\psi$ is isotropic over $F(\varphi)$.

A. Wadsworth in [23] and Knebusch in [16] were among the first to consider the question of when the function fields of two quadratic forms over $F$ are isomorphic. By the above, these two forms must necessarily be of the same dimension, and we also may assume that both forms are anisotropic. Now if the two forms are similar, then clearly their function fields are isomorphic. However, the converse is generally not true. The first counterexamples were given by Knebusch in [17, Example 4.1]. These counterexamples have been generalized by H. Ahmad and J. Ohm [2]. To explain their results, we first recall some definitions. A form of the type $\left\langle 1, a_{1}\right\rangle \otimes \cdots \otimes\left\langle 1, a_{n}\right\rangle$ is called an $n$-fold Pfister form, and we write $\left\langle\left\langle a_{1}, \ldots, a_{n}\right\rangle\right\rangle$ for short. The set of all forms over $F$ isometric (resp. similar) to an $n$-fold Pfister form will be denoted by $P_{n} F$ (resp. $\left.G P_{n} F\right) . \varphi$ is called a Pfister neighbor of the

Received by the editors December 2, 1994 and, in revised form, October 16, 1995.

1991 Mathematics Subject Classification. Primary 11E04, 11E81, 12F20.

Key words and phrases. Quadratic forms of height 2, function fields of quadratic forms, equivalence of function fields, isomorphism of function fields.

This research has been carried out during the author's stay at the Department of Mathematics at the University of Kentucky, Lexington, Kentucky, during the academic year 1994/95.

(C)1996 American Mathematical Society 
Pfister form $\pi$ if $\varphi$ is similar to a subform of $\pi$, i.e. if there exist $a \in \dot{F}=F \backslash\{0\}$ and a form $\psi$ over $F$ such that $\varphi \perp \psi \simeq a \pi$ and $\operatorname{dim} \varphi>\frac{1}{2} \operatorname{dim} \pi$ (we also simply write $\varphi \subset a \pi$ to express the fact that $\varphi$ is isometric to a subform of $a \pi$ ). In [2], a Pfister neighbor $\varphi$ of $\pi \in P_{n} F$ is called special if there exists $\rho \in G P_{n-1} F$ such that $\rho \subset \varphi$. Before we state the result from [2], let us remark that obviously two function fields of quadratic forms are equivalent if they are isomorphic. In [11], the following has been proved.

Theorem 1.1 ([11, Proposition 2]). Let $\varphi$ be a Pfister neighbor of an anisotropic Pfister form $\pi$ over $F$, and let $\psi$ be an anisotropic form over $F$. Then $F(\varphi)$ and $F(\psi)$ are equivalent if and only if $\psi$ is a Pfister neighbor of $\pi$.

It is still an open problem whether the equivalence of two function fields of forms of the same dimension implies that the function fields are isomorphic (see the remarks in section 3 of [21]). For Pfister neighbors, Ahmad and Ohm showed the following result.

Theorem 1.2 ([2, Theorem 1.6, Corollary 2.5]). (i) Let $\varphi$ be a special Pfister neighbor of an anisotropic $\pi \in P_{n} F$ and let $\psi$ be a special Pfister neighbor with $\operatorname{dim} \psi=$ $\operatorname{dim} \varphi$. Then $F(\varphi) \simeq F(\psi)$ iff $\psi$ is a Pfister neighbor of $\pi$.

(ii) If $\varphi$ is a Pfister neighbor of an anisotropic $\pi \in P_{n} F$ with $\operatorname{dim} \varphi \geq 2^{n}-n$, then $\varphi$ is a special Pfister neighbor, and thus, if $\psi$ is a form over $F$ with $\operatorname{dim} \psi=\operatorname{dim} \varphi$, then $F(\varphi) \simeq F(\psi)$ iff $\psi$ is a Pfister neighbor of $\pi$ (and again, $\psi$ is also a special Pfister neighbor).

It is not difficult to construct examples of a field $F$ with an anisotropic 3 -fold Pfister form $\pi$ which contains 5-dimensional Pfister neighbors which are not similar. For instance, let $F=F_{0}((X))((Y))((Z))$ be the iterated Laurent series field in three variables over any field $F_{0}$ of characteristic not 2 , let $\pi \simeq\langle\langle X, Y, Z\rangle\rangle$ and let $\varphi \simeq$ $\langle 1, X, Y, X Y, Z\rangle \subset \pi$ and $\psi \simeq\langle 1, X, Z, X Z, Y\rangle \subset \pi$. By Springer's theorem, it readily follows that $\pi$ is anisotropic and that $\varphi$ and $\psi$ are not similar. However, by the previous theorem, $F(\varphi) \simeq F(\psi)$.

Nevertheless, in some cases isomorphism of the function fields implies similarity of the forms involved. We have the following.

Theorem 1.3. Let $\varphi$ be an anisotropic form over $F$ which is of one of the following types:

- $\varphi$ is a Pfister neighbor of codimension 0 or 1.

- $\operatorname{dim} \varphi=4$ with signed determinant $d_{ \pm} \varphi \neq 1$ (i.e. $\varphi \notin G P_{2} F$ ).

- $\operatorname{dim} \varphi=5$ and $\varphi$ is not a Pfister neighbor.

- $\operatorname{dim} \varphi=6$ and $d_{ \pm} \varphi=1$.

- $\operatorname{dim} \varphi=6, d_{ \pm} \varphi=d \neq 1$ and $\varphi$ is isotropic but not hyperbolic over $F(\sqrt{d})$.

Let $\psi$ be a form over $F$ with $\operatorname{dim} \varphi=\operatorname{dim} \psi$. Then the following statements are equivalent :

(1) $\varphi$ is similar to $\psi$.

(2) $F(\varphi) \simeq F(\psi)$.

(3) $F(\varphi)$ and $F(\psi)$ are equivalent.

$(1) \Rightarrow(2) \Rightarrow(3)$ is obvious. Wadsworth proved the implication $(2) \Rightarrow(1)$ above in the Pfister neighbor case and in the case $\operatorname{dim} \varphi=4$ in [23], however, with only some minor modifications of his arguments one can also show the implication $(3) \Rightarrow(1)$ 
in these cases. A more recent proof of the 4-dimensional case can be found in [21]. Using quite different techniques, D. Leep proved in the case where $\operatorname{dim} \varphi=6$ and $d_{ \pm} \varphi=1$ that if $\varphi$ becomes isotropic over the function field of $\psi$ and $\psi \notin G P_{2} F$, then $\varphi$ contains a subform similar to $\psi$. This readily implies $(3) \Rightarrow(1)$ in the case $\operatorname{dim} \varphi=6$ and $d_{ \pm} \varphi=1$. He also applied these techniques to give yet another proof of the 4-dimensional case ([19]; his proof can also be found in [8]). Using Leep's result, it was shown in [9] that if $\operatorname{dim} \varphi=5$ and $\varphi$ is not a Pfister neighbor, and if $\varphi$ becomes isotropic over the function field of $\psi$ and $\psi \notin G P_{2} F$, then $\varphi$ contains a subform similar to $\psi$. Again, this implies $(3) \Rightarrow(1)$ in this case. Finally, the case $\operatorname{dim} \varphi=6, d_{ \pm} \varphi=d \neq 1$ and $\varphi$ isotropic but not hyperbolic over $F(\sqrt{d})$ follows readily from results in [10].

In this paper, we will extend the above list of forms satisfying the statement in Theorem 1.3. We will show the following theorem.

Theorem 1.4. Let $\varphi$ be an anisotropic form over $F$ of dimension $2^{n}, n \geq 2$, and assume that $\varphi$ can be written $\varphi \simeq \rho \otimes \tau$, where $\rho$ is a Pfister form of fold $n-2$ and $\operatorname{dim} \tau=4$. Let $\psi$ be a form over $F$ of dimension $2^{n}$. Then the following statements are equivalent :

(1) $\varphi$ is similar to $\psi$.

(2) $F(\varphi) \simeq F(\psi)$.

(3) $F(\varphi)$ and $F(\psi)$ are equivalent.

If $\tau$ can be chosen in $G P_{2} F$ then we recover the case where $\varphi$ is similar to an $n$-fold Pfister form. If $n=2$ we recover the 4 -dimensional case. So the results on Pfister forms and 4-dimensional forms in [23] are subsumed in this theorem, and we get new forms of dimension $2^{n}$ which are not similar to Pfister forms where we can decide the question regarding the isomorphism of their function fields. The study of these forms of dimension $2^{n}$ is of interest in its own right. They are forms of height 2 and have been studied by Fitzgerald [7], Kahn [15], and Hurrelbrink and Rehmann [13]. Some of their results and conjectures regarding these forms will be presented in the next section. In fact, by combining Theorems 1.1, 1.2, 1.3, and 1.4 with the classification of forms of height 2 and degree 1 or 2 , we get the following.

Theorem 1.5. Let $\varphi$ be an anisotropic form over $F$ of height 2 and degree 1 or 2. Let $\psi$ be an anisotropic form over $F$ with $\operatorname{dim} \psi=\operatorname{dim} \varphi$. Then the following statements are equivalent :

(1) $F(\varphi) \simeq F(\psi)$.

(2) $F(\varphi)$ and $F(\psi)$ are equivalent.

In section 3, we will prove Theorem 1.4 in a slightly more general setting. We will apply this result in section 4 to derive some consequences and certain generalizations which address the question when a form $\varphi$ of dimension $2^{n}$ of the above type becomes isotropic over a field extension $K$ of $F$, where $K$ is the function field of a single quadratic form or of several $n$-fold Pfister forms. We also extend our results to certain subforms of such a form $\varphi$ of sufficiently small codimension.

The terminology and the notations we use are standard and follow those introduced in Lam's book [18] and Scharlau's book [22]. In the sequel, we list some facts we will frequently use and which can be found in the aforementioned references. If $\pi$ is a Pfister form then $\pi$ is anisotropic or hyperbolic and we have $D_{K}(\pi)=G_{K}(\pi)$ for all field extensions $K / F$, where $D_{K}(\varphi)=\left\{x \in \dot{K} \mid\langle x\rangle \subset \varphi_{K}\right\}$ and $G_{K}(\varphi)=\left\{x \in \dot{K} \mid x \varphi_{K} \simeq \varphi_{K}\right\}$ (we omit the subscript $K$ if $K=F$ ). The 
Cassels-Pfister subform theorem states that if an anisotropic form $\varphi$ becomes hyperbolic over $F(\psi)$, i.e. $\varphi$ lies in the Witt kernel $W(F(\psi) / F)$, then $\varphi$ contains a subform similar to $\psi$. From this, one gets that if $\psi$ is a Pfister form and $\varphi \in W(F(\psi) / F)$ is anisotropic, then there exists $\tau \in W F$ such that $\varphi \simeq \psi \otimes \tau$.

We will need the notions of height, degree, and leading form of a form $\varphi$ as defined in [16] (see also [22, Ch.4, $\S 7]$ ). Let $\varphi \in W F$ and put $\varphi_{0}=\varphi_{\text {an }}$, the anisotropic part of $\varphi$, and $F_{0}=F$. For $i \geq 1$ let $F_{i}=F_{i-1}\left(\varphi_{i-1}\right)$ and $\varphi_{i}=\left(\left(\varphi_{i-1}\right)_{F_{i}}\right)_{\text {an }}$. The smallest number $h$ for which $\operatorname{dim} \varphi_{h} \leq 1$ is called the height of $\varphi$. $\varphi$ is excellent if all $\varphi_{i}, 1 \leq i \leq h$, are defined over $F$ (see [17, Theorem 7.14]). If $\operatorname{dim} \varphi$ is odd the degree of $\varphi, \operatorname{deg} \varphi$, is defined to be 0 , if $\varphi$ is hyperbolic then $\operatorname{deg} \varphi=\infty$, and if $\operatorname{dim} \varphi$ is even and $\varphi$ is not hyperbolic then for some $n, \varphi_{h-1}$ is similar to some $\tau \in P_{n} F_{h-1}$. In this case, one defines $\operatorname{deg} \varphi=n$, and $\tau$ is called the leading form of $\varphi$. If $\tau$ is defined over $F$ then $\varphi$ is said to be a good form. Forms over $F$ of degree $\geq n$ form an ideal $J_{n} F$ in $W F$ which contains the ideal $I^{n} F$, the $n$-th power of the fundamental ideal $I F \subset W F$ of all even-dimensional forms. It is known that for $n \leq 4$ one has $J_{n} F=I^{n} F$ (see [15, Théorème 2.8] for this statement and further references). We will almost exclusively work with the ideals $J_{n} F$. Note that from the definition of $J_{n} F$ it follows immediately that if $\varphi \in J_{n} F$ and $\operatorname{dim} \varphi<2^{n}$ then $\varphi$ is hyperbolic, and if $\varphi \in J_{n} F$ and $\operatorname{dim} \varphi=2^{n}$ then $\varphi \in G P_{n} F$. Note that the result that $I^{n} F \subset J_{n} F$ is equivalent to the Arason-Pfister Hauptsatz which says that if $\varphi \in I^{n} F$ and $\operatorname{dim} \varphi<2^{n}$ then $\varphi$ is hyperbolic.

\section{Some REMARKS ON FORMS OF HeIGHT 2}

In this section, we will have a closer look at forms of the type described in Theorem 1.4, and we try to place them into a larger context. Let us consider the following types of forms.

(1) $\varphi \in W F$ is anisotropic, $\operatorname{dim} \varphi=2^{n+1}$, and there exist $\rho \in P_{n-1} F$ and $\tau \in W F, \operatorname{dim} \tau=4$, such that $\varphi \simeq \rho \otimes \tau$ but $\varphi \notin G P_{n+1} F$.

(2) $\varphi \in W F$ is anisotropic, $\operatorname{dim} \varphi=2^{n+1}$, and there exist an anisotropic $\pi \in$ $P_{n} F$ and an anisotropic Pfister neighbor $\chi$ with $\operatorname{dim} \chi=2^{n}+2^{n-1}$ such that $\varphi \equiv \pi\left(\bmod J_{n+1} F\right)$ and $\chi \subset \varphi$.

(3) $\varphi \in W F$ is anisotropic, $\operatorname{dim} \varphi=2^{n+1}$, and there exists an anisotropic $\pi \in$ $P_{n} F$ such that $\varphi \equiv \pi\left(\bmod J_{n+1} F\right)$.

(4) $\varphi \in W F$ is anisotropic, $\operatorname{dim} \varphi=2^{n+1}$, and $\varphi$ is a good non-excellent form of height 2 and degree $n$.

(5) $\varphi \in W F$ is an anisotropic good non-excellent form of height 2 and degree $n$.

Proposition 2.1. Let $\varphi \in W F$ be anisotropic and let (1) through (5) denote the properties above. Then $(1) \Leftrightarrow(2) \Rightarrow(3) \Leftrightarrow(4) \Rightarrow(5)$.

Proof. $(2) \Rightarrow(3)$ and $(4) \Rightarrow(5)$ are trivial.

$(1) \Rightarrow(2)$. Let $\varphi$ satisfy (1). After scaling if necessary, we may assume that $\tau \simeq\langle d, a, b, a b\rangle$ with $d, a, b \in \dot{F}$. Let $\pi \simeq \rho \otimes\langle 1,-d\rangle$. Clearly, $\pi \in P_{n} F$, and we have $\varphi \perp \pi=\rho \otimes\langle d, a, b, a b, 1,-d\rangle=\rho \otimes\langle\langle a, b\rangle\rangle$ in $W F$. But $\rho \otimes\langle\langle a, b\rangle\rangle \in P_{n+1} F$ and therefore clearly $\varphi \perp \pi \in J_{n+1} F$, or, equivalently, $\varphi \equiv-\pi \equiv \pi\left(\bmod J_{n+1} F\right)$. $\pi$ is anisotropic because otherwise, $\pi$ would be hyperbolic and thus $\varphi \in J_{n+1} F$. But $\operatorname{dim} \varphi=2^{n+1}$ and hence the Arason-Pfister Hauptsatz would imply $\varphi \in G P_{n+1} F$, a case excluded in (1). Finally, the form $\chi \simeq \rho \otimes\langle a, b, a b\rangle \subset \varphi$ is a Pfister neighbor of $\rho \otimes\langle\langle a, b\rangle\rangle$ and $\operatorname{dim} \chi=2^{n}+2^{n-1}$. 
$(2) \Rightarrow(1)$. The case $n=1$ is trivial, so we may assume that $n \geq 2$. Let $\chi, \alpha \in$ $W F$ and $\sigma \in P_{n+1} F$ be such that $\varphi \simeq \chi \perp \alpha$ and such that $\chi$ is a Pfister neighbor of $\sigma$ of dimension $2^{n}+2^{n-1}$. After scaling $\varphi$ (and $\chi$ accordingly) if necessary, we may assume that $\sigma \simeq \chi \perp \beta$ for some $\beta \in W F, \operatorname{dim} \beta=\operatorname{dim} \alpha=2^{n-1}$. Thus,

$$
\varphi \equiv \varphi \perp-\sigma \equiv \alpha \perp-\beta \equiv \pi \quad\left(\bmod J_{n+1} F\right)
$$

It then readily follows from the Arason-Pfister Hauptsatz and the fact that $\pi \in P_{n} F$ and $\operatorname{dim}(\alpha \perp-\beta)=2^{n}$ that $\alpha \perp-\beta$ is in fact similar to $\pi$. Hence, there exists $x \in \dot{F}$ such that $\alpha \perp-\beta \simeq x \pi$, and for the Witt index $i_{W}$ we get $i_{W}(\sigma \perp x \pi)$ $\geq \operatorname{dim} \beta=2^{n-1}$. By [3, Proposition 4.6] there exist Pfister forms $\rho \in P_{n-1} F$, $\eta \in P_{2} F$, and $r \in \dot{F}$ such that $\sigma \simeq \rho \otimes \eta$ and $\pi \simeq \rho \otimes\langle\langle r\rangle\rangle$. (This result in [3] is only stated for linkage of two Pfister forms of the same dimension, but it also applies to two Pfister forms of different dimension. The proof carries over without change.) By assumption, $\operatorname{dim} \rho \geq 2$. Let $K=F(\rho)$. Then $\rho_{K}=0$ in $W K$ and therefore $\sigma_{K}=\pi_{K}=0$ in $W K$ as well. Hence, $\chi_{K}$ is isotropic and thus $\operatorname{dim}\left(\varphi_{K}\right)_{\text {an }}<\operatorname{dim} \varphi=2^{n+1}$. On the other hand, $\left(\varphi_{K}\right)_{\text {an }} \equiv \pi_{K} \equiv 0\left(\bmod J_{n+1} K\right)$. The Arason-Pfister Hauptsatz then implies that $\varphi_{K}=0$ in $W K$, i.e. $\varphi$ is an anisotropic form in $W(F(\rho) / F)$. Hence, by the Cassels-Pfister subform theorem there exists $\tau \in W F$ with $\varphi \simeq \rho \otimes \tau$. Comparing dimensions yields $\operatorname{dim} \tau=4$. Also, $\varphi \notin G P_{n+1} F$ because $\varphi \equiv \pi \not \equiv 0\left(\bmod J_{n+1} F\right)$.

$(3) \Rightarrow(4)$. Let $L=F(\varphi)$. Clearly, $\pi_{L} \in P_{n} L$ is not hyperbolic because $\varphi$ is not similar to a subform of $\pi$ for dimension reasons and by the Cassels-Pfister subform theorem. Hence, $\pi_{L}$ is anisotropic and we have $\left(\varphi_{L}\right)_{\text {an }} \equiv \pi_{L} \not \equiv 0\left(\bmod J_{n+1} L\right)$. In particular, $\varphi_{L}$ is not hyperbolic and we have $0<\operatorname{dim}\left(\varphi_{L}\right)$ an $<2^{n+1}=\operatorname{dim} \varphi$. Now over $L(\pi)$ we have $\varphi_{L(\pi)} \equiv \pi_{L(\pi)} \equiv 0\left(\bmod J_{n+1} L(\pi)\right)$ and $\operatorname{dim}\left(\varphi_{L(\pi)}\right)$ an $<2^{n+1}$. By the Arason-Pfister Hauptsatz we get $\varphi_{L(\pi)}=0$ in $W L(\pi)$. Hence, there exists a form $\alpha \in W L$ such that $\left(\varphi_{L}\right)_{\text {an }} \simeq \alpha \otimes \pi_{L}$. Comparing dimensions, we must have $\operatorname{dim} \alpha=1$, which implies that $\left(\varphi_{L}\right)_{\text {an }}$ is similar to $\pi_{L}$. We readily conclude that $\varphi$ has height 2 and leading form $\pi_{L} \in P_{n} L$ which is obviously defined over $F$. Hence, $\varphi$ is good of height 2 and degree $n$. Furthermore, $\varphi$ is not excellent because $\varphi \notin G P_{n+1} F$ and the only excellent forms of dimension $2^{n+1}$ are anisotropic forms in $G P_{n+1} F$.

$(4) \Rightarrow(3)$. Cf. $[17$, Theorem 9.6].

In [17, Lemma 10.1] it is shown that forms fulfilling (5), i.e. good non-excellent forms of height 2 and degree $n$, must have dimension $2^{N}$ with $N \geq n+1$. Thus, $(5) \Rightarrow(4)$ means that $N=n+1$. Now it is not known whether $(5) \Rightarrow$ (4) for all $n$, nor do we know whether $(3) \Rightarrow(2)$. We have the following conjecture which in essence is due to Fitzgerald [7, 1.3] and which has been restated by B. Kahn in [15, Conjecture $7 \mathrm{a}]$.

Conjecture 2.2. (5) $\Rightarrow(1)$, i.e. the statements (1) through (5) are equivalent.

In [13, Section 3] it was shown that if certain conjectures on higher cohomological invariants are true then $(5) \Rightarrow(4)$ (more explicitly, if these conjectures are true and $\varphi$ fulfills (5) then there exists a field extension $K / F, \rho \in P_{n-1} K, \tau \in W K$, $\operatorname{dim} \tau=4$, such that $\varphi_{K}$ is anisotropic and $\left.\varphi_{K} \simeq \rho \otimes \tau\right)$. We do have the following definite result.

Theorem 2.3. If $n \leq 3$ then $(5) \Rightarrow(1)$, i.e. Conjecture 2.2 is true and the statements (1) through (5) are equivalent. 
The case $n=1$ is due to Knebusch [17, Theorem 10.3], the case $n=2$ was proved by Fitzgerald in [7, Theorem 1.6]. The implication $(5) \Rightarrow(4)$ for $n=3$ was proved in [13, Theorem 3.4], and Rost proved $(3) \Rightarrow(1)$ in this case (see also Kahn's paper [15, Théorèmes 2.12, 4.2, 4.3]). Rost's (still unpublished) result and his own proof thereof were communicated to me by Kahn and will be included in a forthcoming paper by the present author.

If Conjecture 2.2 is true then Theorem 1.4 just says that if $\varphi$ and $\psi$ are forms over $F$ and if $\varphi$ is anisotropic, good and non-excellent of height 2, then $F(\varphi) \simeq F(\psi)$ if and only if $\psi$ is similar to $\varphi$.

\section{Proof of the main Result}

Let us first start with a preliminary lemma which states some useful facts.

Lemma 3.1. Let $m>n \geq 1$ and let $\varphi \in W F$ be anisotropic and $\operatorname{dim} \varphi=2^{m}$, and let $\pi \in G P_{n} F$ be anisotropic. Suppose that $\varphi \equiv \pi\left(\bmod J_{m} F\right)$. Let $E=F(\pi)$. Then the following hold.

(1) $\varphi_{E}$ is anisotropic and in $G P_{m} E$.

(2) If $\psi \in W F, \operatorname{dim} \psi \geq 2$, and if $\varphi_{F(\psi)}$ is isotropic, then $\psi_{E}$ is similar to a subform of $\varphi_{E}$.

(3) If $\psi \in W F$, $\operatorname{dim} \psi=2^{m}$, and if $\psi_{E}$ is similar to $\varphi_{E}$, then $\operatorname{deg} \psi \geq n$. If furthermore $\operatorname{deg} \psi=n$ then $\psi \equiv \pi\left(\bmod J_{n+1} F\right)$. In particular, if $\varphi_{F(\psi)}$ is isotropic then $\operatorname{deg} \psi \geq n$, and if furthermore $\operatorname{deg} \psi=n$ then $\psi \equiv \pi$ $\left(\bmod J_{n+1} F\right)$.

Proof. (1) Clearly, $\pi_{E}=0$ in $W E$ and therefore $\varphi_{E} \in J_{m} E$. As $\operatorname{dim} \varphi=2^{m}$, the Arason-Pfister Hauptsatz implies that $\varphi_{E}$ is either similar to an anisotropic $m$-fold Pfister form over $E$, in which case we are done, or $\varphi_{E}$ is hyperbolic. In the latter case, there exists a form $\tau \in W F$ such that $\varphi \simeq \pi \otimes \tau$, because $\varphi$ is an anisotropic form in $W(F(\pi) / F)$. But then $\operatorname{dim} \tau=2^{n}-2^{m}$, which is even. We conclude that $\varphi \simeq \pi \otimes \tau \in J_{n+1} F$, because $\tau \in I F$ and $\pi \in J_{n} F$ (see [16, Proposition 6.9]), a contradiction to $\varphi \equiv \pi \not \equiv 0\left(\bmod J_{n+1} F\right)$.

(2) By part (1), $\varphi_{E} \in G P_{m} E$ is anisotropic. Hence, if $\varphi_{F(\psi)}$ is isotropic then $\varphi_{E(\psi)}$ is obviously isotropic as well, and therefore hyperbolic. It follows immediately from the Cassels-Pfister subform theorem that $\psi_{E}$ is similar to a subform of $\varphi_{E}$.

(3) Let $r=\operatorname{deg} \psi$. Clearly, $r \leq m$ because $\operatorname{dim} \psi=2^{m}$. Now $\psi_{E}$ is similar to $\varphi_{E}$, which is in $G P_{m} E$. Hence, $\psi_{E} \in G P_{m} E$, and therefore $\operatorname{deg} \psi_{E}=m>n$. Since $m>n$ and since $E=F(\pi)$ with $\operatorname{dim} \pi=2^{n}$, it follows from [1, Satz 18] that $\operatorname{deg} \psi \geq n$, and that if $\operatorname{deg} \psi=n$ then $\psi \equiv \pi\left(\bmod J_{n+1} F\right)$. The remaining statement follows from this together with part (2).

In the proof of our first main result we will need the following lemma.

Lemma 3.2. Let $\alpha$ and $\beta$ be Pfister neighbors of $\pi \in P_{n+1} F, n \geq 1$, and suppose that $\operatorname{dim} \alpha, \operatorname{dim} \beta \geq 2^{n}+2^{n-1}$. Then there exist $\chi \subset \pi$ with $\operatorname{dim} \chi=2^{n}+1$, and $x, y \in \dot{F}$ such that $x \chi \subset \alpha$ and $y \chi \subset \beta$, i.e. $x \alpha$ and $y \beta$ have a Pfister neighbor of $\pi$ as a common subform.

Proof. Without loss of generality, we may assume $\operatorname{dim} \alpha=\operatorname{dim} \beta=2^{n}+2^{n-1}$. After scaling if necessary, we may further assume that $\alpha \subset \pi$ and $\beta \subset \pi$. Hence, there are $x, y \in \dot{F}$ and $\mu, \eta \in W F$ with $\operatorname{dim} \mu=\operatorname{dim} \eta=2^{n-1}-1$ such that

$$
\pi \simeq \alpha \perp \mu \perp\langle x\rangle \simeq \beta \perp \eta \perp\langle y\rangle \text {. }
$$


Let $\pi \simeq\langle 1\rangle \perp \pi^{\prime}$. We have $x, y \in D(\pi)=G(\pi)$ and thus $x \pi \simeq \pi \simeq y \pi$. Therefore,

$$
\langle 1\rangle \perp \pi^{\prime} \simeq x \alpha \perp x \mu \perp\langle 1\rangle \simeq y \beta \perp y \eta \perp\langle 1\rangle
$$

which yields $x \alpha \perp x \mu \simeq y \beta \perp y \eta$. By Witt cancellation, we get

$$
x \alpha \perp-y \beta \perp\left(2^{n-1}-1\right) \mathbb{H} \simeq y \eta \perp-x \mu \perp\left(2^{n}+2^{n-1}\right) \mathbb{H}
$$

which implies, after cancelling hyperbolic planes, that the Witt index of $x \alpha \perp-y \beta$ is at least $2^{n}+1$. In particular, there exists $\chi \in W F$ with $\operatorname{dim} \chi=2^{n}+1$ such that $\chi \subset x \alpha$ and $\chi \subset y \beta$. Clearly, $\chi$ is a Pfister neighbor of $\pi$.

We now generalize Wadsworth's result on 4-dimensional forms [23, Theorem 7]. His result is obtained by putting $n=1$ in the theorem below. B. Kahn informed me that a student of his, A. Laghribi, independently obtained results which amount to the theorem below in the case $n=2$.

Theorem 3.3. Let $\varphi$ be anisotropic, $\operatorname{dim} \varphi=2^{n+1}$ and $\varphi \equiv \pi\left(\bmod J_{n+1} F\right)$ for some anisotropic $\pi \in P_{n} F$. Let $\psi$ be anisotropic with $\operatorname{dim} \psi=2^{n+1}$. Suppose in addition that if $n \geq 4$ then $\psi$ contains a Pfister neighbor of dimension $2^{n}+2^{n-1}$. Let $E=F(\pi)$. Then the following statements are equivalent:

(1) $\psi_{E}$ is similar to $\varphi_{E}$.

(2) Either $\psi$ is similar to some $\gamma \in P_{n+1} F$ and $\varphi$ contains a Pfister neighbor of $\gamma$ of dimension $2^{n}+2^{n-1}$, or $\psi$ is similar to $\varphi$.

(3) $\varphi_{F(\psi)}$ is isotropic.

In particular, if these equivalent statements hold then there exist $\rho \in P_{n-1} F$ and $\tau \in W F, \operatorname{dim} \tau=4$, such that $\varphi \simeq \rho \otimes \tau$.

Proof. (3) $\Rightarrow$ (1) follows immediately from Lemma 3.1(2). Now if $\psi$ is similar to $\varphi$ then clearly $\varphi_{F(\psi)}$ is isotropic. Also, if $\psi$ is similar to some $\gamma \in P_{n+1} F$ and if $\chi$ is a Pfister neighbor of $\gamma$ and simultaneously a subform of $\varphi$, then obviously $\chi_{F(\psi)}$ is isotropic, and therefore so is $\varphi_{F(\psi)}$. This proves $(2) \Rightarrow(3)$.

It remains to prove that (1) implies (2). We may assume that $1 \in D(\varphi)$ and $1 \in D(\psi)$. First, let us consider the case $\psi \in P_{n+1} F$. By Lemma 3.1 we have $\varphi_{E} \in$ $P_{n+1} E$ and thus, by assumption and the fact that $1 \in D_{E}\left(\varphi_{E}\right)$ and $1 \in G_{E}\left(\psi_{E}\right)$, we get $\varphi_{E} \simeq \psi_{E}$. In particular, $\varphi \perp-\psi \in W(E / F)=W(F(\pi) / F)$ and there exists $\sigma \in W F$ such that $(\varphi \perp-\psi)_{\text {an }} \simeq \sigma \otimes \pi$. Note that $\operatorname{dim}(\varphi \perp-\psi)_{\text {an }} \leq 2^{n+2}-2$ because both $\varphi$ and $\psi$ represent 1 , and that

$$
\varphi \perp-\psi \equiv \varphi \equiv \pi \not \equiv 0 \quad\left(\bmod J_{n+1} F\right) .
$$

Suppose $\operatorname{dim} \sigma$ is even, i.e. $\sigma \in I F$. Then $\varphi \perp-\psi=\sigma \otimes \pi \in J_{n+1} F$ because $\pi \in P_{n} F$ and by [16, Proposition 6.9] (or [22, Theorem 7.5]), a contradiction. Hence, $\operatorname{dim} \sigma$ is odd, which leaves as the only possibilities $\operatorname{dim} \sigma=1$ or $\operatorname{dim} \sigma=3$. If $\operatorname{dim} \sigma=1$ then by comparing $\operatorname{dim}(\varphi \perp-\psi)=2^{n+2}$ with $\operatorname{dim}(\varphi \perp-\psi)_{\text {an }}=$ $\operatorname{dim} \pi=2^{n}$, we see that the Witt index of $\varphi \perp-\psi$ is just $\frac{1}{2}\left(2^{n+2}-2^{n}\right)=2^{n}+2^{n-1}$. This implies that there exists a Pfister neighbor $\alpha \subset \psi$ with $\operatorname{dim} \alpha=2^{n}+2^{n-1}$ such that $\alpha \subset \varphi$, and we are done in this case.

Now if $\operatorname{dim} \sigma=3$, say, $\sigma \simeq r\langle s, t, s t\rangle, r, s, t \in \dot{F}$, then

$$
(\varphi \perp-\psi)_{\text {an }} \perp r \pi \simeq \sigma \otimes \pi \perp r \pi \simeq r \pi \otimes\langle\langle s, t\rangle\rangle \in G P_{n+2} F .
$$

In particular, $(\varphi \perp-\psi)_{\text {an }}$ is an anisotropic Pfister neighbor of $\pi \otimes\langle\langle s, t\rangle\rangle \in$ $P_{n+2} F$ which is therefore itself anisotropic. Now over $F(\psi)$ we have that 
$\left((\varphi \perp-\psi)_{\text {an }}\right)_{F(\psi)}=\varphi_{F(\psi)}$ in $W F(\psi)$. Comparing dimensions, we see that the Pfister neighbor $(\varphi \perp-\psi)$ an becomes isotropic over $F(\psi)$, and that therefore $\pi \otimes\langle\langle s, t\rangle\rangle$ becomes hyperbolic over $F(\psi)$. As $\psi \in P_{n+1} F$, there are $a, b \in \dot{F}$ such that

$$
(\varphi \perp-\psi)_{\text {an }} \perp r \pi \simeq r \pi \otimes\langle\langle s, t\rangle\rangle \simeq \psi \otimes\langle a, b\rangle .
$$

Now for suitable subforms $\varphi^{\prime} \subset \varphi$ and $\psi^{\prime} \subset \psi$ with $\operatorname{dim} \varphi^{\prime}=\operatorname{dim} \psi^{\prime}=2^{n}+2^{n-1}$ we have $(\varphi \perp-\psi)_{\text {an }} \simeq \varphi^{\prime} \perp-\psi^{\prime}$. In particular, we may assume that already $a \in D\left(-\psi^{\prime}\right) \subset D(-\psi)$, i.e. $-a \in D(\psi)=G(\psi)$. It follows that

$$
\varphi \perp-\psi \perp r \pi=a \psi \perp b \psi=-\psi \perp b \psi \text { in } W F
$$

which, by Witt cancellation, yields that $\varphi \perp-b \psi=-r \pi$ in $W F$. By comparing dimensions, we conclude, similarly as above, that there exist $\alpha \subset b \psi$ with $\operatorname{dim} \alpha=$ $2^{n}+2^{n-1}$ such that $\alpha \subset \varphi$.

Let us finally consider the case $\psi \notin P_{n+1} F$. In particular, this implies that $\operatorname{deg} \psi \leq n$. Since $\varphi_{F(\psi)}$ is isotropic, Lemma 3.1(3) yields that $\psi \equiv \pi\left(\bmod J_{n+1} F\right)$. We conclude that there exists $\beta \subset \psi$ with $\operatorname{dim} \beta=2^{n}+2^{n-1}$ such that $\beta$ is a Pfister neighbor of some (anisotropic) $\tilde{\psi} \in P_{n+1} F$. For $n \geq 4$ this follows from our assumption on $\psi$, and for $n \leq 3$ this follows from Theorem 2.3. As $\varphi_{E} \in P_{n+1} E$ is similar to $\psi_{E}$ and thus clearly contains a subform similar to $\beta_{E}$, we readily conclude that $\varphi_{E} \simeq \tilde{\psi}_{E}$. By the previous case, there exists a Pfister neighbor $\alpha$ of $\tilde{\psi}$ with $\operatorname{dim} \alpha=2^{n}+2^{n-1}$ such that $\alpha \subset \varphi$. By Lemma 3.2, there exist $x, y \in \dot{F}$ and $\chi \in W F$ with $\operatorname{dim} \chi=2^{n}+1$ such that $\chi \subset x \alpha \subset x \varphi$ and $\chi \subset y \beta \subset y \psi$. In particular, the Witt index of $x \varphi \perp-y \psi$ is at least $2^{n}+1=\operatorname{dim} \chi$. Hence, $\operatorname{dim}(x \varphi \perp-y \psi)_{\text {an }} \leq 2^{n+2}-2\left(2^{n}+1\right)<2^{n+1}$. Recall that we have $\varphi \equiv \psi \equiv \pi$ $\left(\bmod J_{n+1} F\right)$. Clearly, $\pi \equiv x \pi \equiv y \pi\left(\bmod J_{n+1} F\right)$. Therefore,

$$
(x \varphi \perp-y \psi)_{\text {an }} \equiv x \varphi \perp-y \psi \equiv x \pi \perp-y \pi \equiv 0 \quad\left(\bmod J_{n+1} F\right) .
$$

Since $(x \varphi \perp-y \psi)_{\text {an }} \in J_{n+1} F$ and $\operatorname{dim}(x \varphi \perp-y \psi)_{\text {an }}<2^{n+1}$, it follows that $x \varphi \perp-y \psi=0$ in $W F$, i.e. $x \varphi \simeq y \psi$.

Now if the two equivalent statements of the theorem hold, then $\varphi$ contains a Pfister neighbor of dimension $2^{n}+2^{n-1}$. By Proposition 2.1 it follows that there exist $\rho \in P_{n-1} F$ and $\tau \in W F, \operatorname{dim} \tau=4$, such that $\varphi \simeq \rho \otimes \tau$ and the theorem is proved in full.

Note that the last statement of the theorem always holds if $n \leq 3$, as follows immediately from Theorem 2.3. In fact, if Conjecture 2.2 holds for $n$ (as it does if $n \leq 3$ ), then we can remove altogether the assumption that $\psi$ contains a Pfister neighbor of dimension $2^{n}+2^{n-1}$, and the theorem will still be valid.

As a consequence of the previous theorem we get Theorem 1.4, which we restate below as a corollary and in slightly different form. Again, if Conjecture 2.2 holds for $n$ we may drop the assumption that $\psi$ or $\varphi$ contains a Pfister neighbor of dimension $2^{n}+2^{n-1}$.

Corollary 3.4. Let $\varphi, \psi \in W F$ be anisotropic with $\operatorname{dim} \varphi=\operatorname{dim} \psi=2^{n+1}$. Let $\pi \in P_{n} F$ be anisotropic. Suppose that $\varphi$ or $\psi$ is equivalent to $\pi\left(\bmod J_{n+1} F\right)$, and that if $n \geq 4$ then $\varphi$ or $\psi$ contains a Pfister neighbor of dimension $2^{n}+2^{n-1}$. Then the following statements are equivalent:

(1) $\varphi$ is similar to $\psi$.

(2) $F(\varphi) \simeq F(\psi)$.

(3) $F(\varphi)$ and $F(\psi)$ are equivalent. 
In particular, if these equivalent statements hold then there exist $\rho \in P_{n-1} F$ and $\tau \in W F, \operatorname{dim} \tau=4$, such that $\varphi$ and $\psi$ are similar to $\rho \otimes \tau$.

Proof. It suffices to prove $(3) \Rightarrow(1)$. We may assume that $\varphi \equiv \pi\left(\bmod J_{n+1} F\right)$. Since $\varphi_{F(\psi)}$ is isotropic it follows from Lemma 3.1(3) that $\operatorname{deg} \psi \geq n$. Clearly, $\operatorname{deg} \psi \leq n+1$ because $\operatorname{dim} \psi=2^{n+1}$. Now if $\operatorname{deg} \psi=n+1$, then $\psi \in J_{n+1} F$ and it follows that $\psi \in G P_{n+1} F$. Obviously, $\varphi$ is not a Pfister neighbor of $\psi$ and therefore $F(\psi)$ and $F(\varphi)$ are not equivalent, a contradiction. Hence, $\operatorname{deg} \psi=n$, and Lemma 3.1(3) implies that $\psi \equiv \varphi \equiv \pi\left(\bmod J_{n+1} F\right)$. Now $F(\varphi)$ and $F(\psi)$ being equivalent just means that $\varphi_{F(\psi)}$ and $\psi_{F(\varphi)}$ are both isotropic. We also know that $\varphi$ or $\psi$ contains a Pfister neighbor of dimension $2^{n}+2^{n-1}$ (by assumption if $n \geq 4$, and by Theorem 2.3 if $n \leq 3$ ). It follows from Theorem 3.3 that there exist $\rho \in P_{n-1} F$ and $\tau \in W F, \operatorname{dim} \tau=4$, such that $\varphi$ and $\psi$ are similar to $\rho \otimes \tau$.

We remark that if a priori we have that, say, $\varphi \simeq \rho \otimes \tau$ with $\rho \in P_{n-1} F$ and $\operatorname{dim} \tau=4$ such that $\varphi \notin G P_{n+1} F$, then the hypotheses on $\varphi$ and $\psi$ in the above corollary are automatically fulfilled by Proposition 2.1 provided $\varphi$ and $\psi$ are anisotropic and $\operatorname{dim} \psi=2^{n+1}$. We then recover the statement of Theorem 1.4.

As another consequence we get Theorem 1.5, which we also restate as a corollary. The proof uses the classification of forms of height 2 and degree 1 by Knebusch, and of forms of height 2 and degree 2 due in part to Fitzgerald and later completed by Kahn.

Corollary 3.5. Let $\varphi$ be an anisotropic form over $F$ of height 2 and degree 1 or 2. Let $\psi$ be an anisotropic form over $F$ with $\operatorname{dim} \psi=\operatorname{dim} \varphi$. Then the following statements are equivalent :

(1) $F(\varphi) \simeq F(\psi)$.

(2) $F(\varphi)$ and $F(\psi)$ are equivalent.

Proof. The only anisotropic forms $\varphi$ of height 2 and degree 1 are either 4-dimensional forms $\varphi$ with $d_{ \pm} \varphi \neq 1$, or Pfister neighbors of dimension $2^{n}-2$ for $n \geq 3$ (see [17, Theorem 10.3]). Note that these Pfister neighbors are always special (cf. Theorem 3.2). The statement of the corollary in the case of height 2 and degree 1 follows immediately from Theorems 1.1, 1.2, and 1.3.

Now let $\varphi$ be an anisotropic form of height 2 and degree 2. The only excellent forms of this type are Pfister neighbors in $I^{2} F$ of dimension $2^{n}-4, n \geq 4$ (see [7, Theorem 1.6] or [15, Lemme 2.2]), which are special by Theorem 1.2. So the assertion in the case of excellent forms of height and degree 2 follows from Theorems 1.1 and 1.2. Now if $\varphi$ is good but not excellent, then by Theorem 2.3 (see also [7, Theorem 1.6] or [15, Corollaire 2.1]), there exist $\rho \in P_{1} F$ and $\tau \in W F, \operatorname{dim} \tau=$ 4 , such that $\varphi \simeq \rho \otimes \tau$. In this case, the assertion follows from the previous corollary (resp. Theorem 1.4). Finally, if $\varphi$ is not $\operatorname{good}$ then $\operatorname{dim} \varphi=6$ and $d_{ \pm} \varphi=1 \in \dot{F} / \dot{F}^{2}$ (cf. [15, Théorème 2.11]), and in this case the corollary follows from Theorem 1.3.

\section{Some FURTHER CONJECTURES AND RESUlTS}

(a) Isotropy of forms of height 2 over the function field of a quadratic form. An interesting question in the algebraic theory of quadratic forms is what are the forms $\psi, \operatorname{dim} \psi \geq 2$, such that a given anisotropic form $\varphi \in W F$ becomes isotropic over $F(\psi)$. The answer is easy if $\operatorname{dim} \psi=2$ because in this case $F(\psi)$ is 
just a quadratic extension of $F$, and $\varphi_{F(\psi)}$ is isotropic iff $\psi$ is similar to a subform of $\varphi$ (cf. [18, Ch.VII, Lemma 3.1] or [22, Ch.2, Lemma 5.1]). The answer is also known if $\varphi$ is a Pfister neighbor, say, of $\gamma \in P F$. Then $\varphi_{F(\psi)}$ is isotropic iff $\psi$ is similar to a subform of $\gamma$. This is an easy consequence of the Cassels-Pfister subform theorem. As mentioned in the introduction, the above problem is fully solved if $\operatorname{dim} \varphi \leq 5$, and there are some partial results for $\operatorname{dim} \varphi=6$.

Before we state our next theorem, let us recall that a field extension $K / F$ is called excellent if for any form $\varphi \in W F$ there exists a form $\eta \in W F$ such that $\left(\varphi_{K}\right)_{\text {an }} \simeq \eta_{K}$, i.e. the anisotropic part of $\varphi$ over $K$ is defined already over $F$. Knebusch has shown that if $F(\psi) / F$ is excellent where $\psi \in W F$ is anisotropic, then $\psi$ is a Pfister neighbor (see [17, Theorem 7.13]). As for the converse, it suffices to consider Pfister forms. It has been an open problem for quite some time whether $F(\psi) / F$ is excellent for all Pfister forms $\psi$. The answer is yes if $\psi$ is of fold $n \leq 2$ (the case $n=1$, i.e. $\operatorname{dim} \psi=2$, follows readily from the remarks above, and the case $n=2$ is due to Arason [5, Appendix II]). If $n \geq 3$ the answer is generally no, as was recently shown by Izhboldin [14]. However, it is known to be yes for certain types of fields $F$, for example for linked fields. The case $n=2$ in the following theorem has been proved by A. Laghribi and was communicated to me by B. Kahn.

Theorem 4.1. Let $\varphi, \pi \in W F$ be anisotropic with $\operatorname{dim} \varphi=2^{n+1}$ and $\pi \in P_{n} F$. Suppose that $\varphi \equiv \pi\left(\bmod J_{n+1} F\right)$. Suppose further that $F(\pi) / F$ is an excellent field extension (which always holds if $n=1,2$ ). Let $\psi \in W F$ with $\operatorname{dim} \psi \geq 2$. Then the following statements are equivalent:

(1) $\varphi_{F(\psi)}$ is isotropic.

(2) $\psi$ is similar to a subform of $\varphi$, or $\psi$ is similar to a subform of some $\gamma \in$ $P_{n+1} F$ and $\varphi$ contains a Pfister neighbor of $\gamma$.

Proof. It is straightforward that (2) implies (1), and we leave the details to the reader. So let us assume that $\varphi_{F(\psi)}$ is isotropic. Let $E=F(\pi)$. By Lemma 3.1, parts (1) and (2), we have that $\psi_{E}$ is similar to a subform of the anisotropic form $\varphi_{E} \in G P_{n+1} E$. After scaling if necessary, we may assume that both $\varphi$ and $\psi$ represent 1 over $F$. It readily follows that then we have $\psi_{E} \subset \varphi_{E} \in P_{n+1} E$. Since $E / F$ is excellent, there exists $\eta \in W F$ such that $\psi_{E} \perp \eta_{E} \simeq \varphi_{E}$. Consider the form $\mu \simeq \psi \perp \eta \in W F$. Now $\mu_{E} \simeq \varphi_{E}$ and thus, by Lemma 3.1(3), we have that either $\operatorname{deg} \mu=n$ and $\mu \equiv \pi\left(\bmod J_{n+1} F\right)$, or $\operatorname{deg} \mu=n+1$ and thus $\mu \in P_{n+1} F$ (note that $\mu$ represents 1 ). In the latter case, $\varphi$ contains a Pfister neighbor of $\mu$ of dimension $2^{n}+2^{n-1}$ by Theorem 3.3. So let us assume that $\mu \equiv \pi$ $\left(\bmod J_{n+1} F\right)$ and $\mu_{E} \simeq \varphi_{E}$. Note that $\mu$ is a good non-excellent form of height 2 and degree $n$ by Proposition 2.1. Since $E / F$ is excellent, it follows from the proof of [7, Theorem 1.6] that there exist $\rho \in P_{n-1} F$ and $\tau \in W F, \operatorname{dim} \tau=4$, such that $\mu \simeq \rho \otimes \tau$, in particular, $\mu$ contains a Pfister neighbor of dimension $2^{n}+2^{n-1}$, again by Proposition 2.1. By invoking Theorem 3.3, we conclude that $\varphi$ is similar to $\mu$ because $\varphi_{E} \simeq \mu_{E}$ and $\mu$ is not in $G P_{n+1} F$. In particular, $\psi$ is similar to a subform of $\varphi$.

It seems natural to ask whether the above also holds without explicitly assuming excellence. 
Conjecture 4.2. Let $\varphi, \pi \in W F$ be anisotropic with $\operatorname{dim} \varphi=2^{n+1}$ and $\pi \in P_{n} F$. Suppose that $\varphi \equiv \pi\left(\bmod J_{n+1} F\right)$. Let $\psi \in W F$ with $\operatorname{dim} \psi \geq 2$. Then the following statements are equivalent:

(1) $\varphi_{F(\psi)}$ is isotropic.

(2) $\psi$ is similar to a subform of $\varphi$, or $\psi$ is similar to a subform of some $\gamma \in$ $P_{n+1} F$ and $\varphi$ contains a Pfister neighbor of $\gamma$.

(b) Equivalence of function fields. Let us now turn to the problem of determining those forms $\psi$ for which $F(\psi)$ is equivalent to $F(\varphi)$ where $\varphi$ is given as in the previous conjecture. This leads to another conjecture which we now state.

Conjecture 4.3. Let $\varphi, \pi \in W F$ be anisotropic with $\operatorname{dim} \varphi=2^{n+1}$ and $\pi \in P_{n} F$. Suppose that $\varphi \equiv \pi\left(\bmod J_{n+1} F\right)$. Let $\psi \in W F$. Then the following statements are equivalent:

(1) $F(\psi)$ is equivalent to $F(\varphi)$.

(2) $\psi$ is similar to a subform of $\varphi$ and $\operatorname{dim} \psi>2^{n}+2^{n-1}$.

Theorem 4.4. In Conjecture 4.3, (2) $\Rightarrow(1)$ holds for any $n$. In particular, any form $\psi$ which is similar to a subform of $\varphi$ and with $\operatorname{dim} \psi>2^{n}+2^{n-1}$ is not a Pfister neighbor.

(1) $\Rightarrow(2)$ holds for $n=1,2$. In particular, Conjecture 4.3 is true for $n=1,2$.

Proof. Let $\varphi, \pi \in W F$ be anisotropic with $\operatorname{dim} \varphi=2^{n+1}, \pi \in P_{n} F$, and $\varphi \equiv \pi$ $\left(\bmod J_{n+1} F\right)$. Since $\varphi$ has height 2 and degree $n$ (see Proposition 2.1), it is clear that $\operatorname{dim}\left(\varphi_{F(\varphi)}\right)$ an $=2^{n}$, i.e. the Witt index of $\varphi$ over $F(\varphi)$ is exactly $2^{n-1}$. This readily implies that any subform $\psi$ of $\varphi$ with $\operatorname{dim} \psi \geq 2^{n+1}-2^{n-1}+1>2^{n}+2^{n-1}$ becomes isotropic over $F(\varphi)$. On the other hand, for any such subform $\psi$ of $\varphi$ we obviously have that $\varphi_{F(\psi)}$ is isotropic. Hence, if $\psi$ is similar to a subform of $\varphi$ and $\operatorname{dim} \psi>2^{n}+2^{n-1}$, then $\psi_{F(\varphi)}$ and $\varphi_{F(\psi)}$ are isotropic and $F(\varphi)$ and $F(\psi)$ are equivalent. Thus $(2) \Rightarrow(1)$. Since $\varphi$ is not a Pfister neighbor (i.e. $\varphi \notin G P_{n+1} F$ ), it follows from Theorem 1.1 that $\psi$ is not a Pfister neighbor.

Now let $n=1,2$, and let $\psi \in W F$ be anisotropic such that $F(\psi)$ and $F(\varphi)$ are equivalent. This implies that $\psi_{F(\varphi)}$ is isotropic. By [11, Theorem 1], we must have $\operatorname{dim} \psi>2^{n}$. We also have that $\varphi_{F(\psi)}$ is isotropic. By Theorem 4.1, we conclude that $\psi$ is a Pfister neighbor of some $(n+1)$-fold Pfister form or $\psi$ is similar to a subform of $\varphi$. As $\varphi$ itself is not a Pfister neighbor, it follows from Theorem 1.1 that $\psi$ is not a Pfister neighbor. Hence, $\psi$ must be similar to a subform of $\varphi$ and $\psi$ must not be a Pfister neighbor.

If $n=1$ then $\operatorname{dim} \psi=3$ or 4 . But any 3 -dimensional form is a Pfister neighbor, so by the above we must have $\operatorname{dim} \psi=4>2^{n}+2^{n-1}=3$ for $n=1$, as desired.

If $n=2$ then $\operatorname{dim} \psi \geq 5$. Now it follows from [9, Main Theorem] and [10, Theorem 2, Theorem 3] that if an anisotropic form $\alpha$ of dimension 5 or 6 becomes isotropic over the function field of a form $\beta$ of dimension 7 or 8 , then $\beta$ is a Pfister neighbor of some 3 -fold Pfister form. Hence, as $\varphi$ is not a Pfister neighbor, we must have $\operatorname{dim} \psi \geq 7>2^{n}+2^{n-1}=6$ for $n=2$, as desired.

(c) Forms of height 2 under Pfister extensions. A Pfister extension $K$ of the field $F$ is just a function field of the kind $K=F\left(\left\{\psi_{i}\right\}\right)$, where $\left\{\psi_{i}\right\}$ is an indexed set of Pfister forms. Let $\varphi, \pi \in W F$ be anisotropic with $\operatorname{dim} \varphi=2^{n+1}, \pi \in P_{n} F$, and $\varphi \equiv \pi\left(\bmod J_{n+1} F\right)$. For certain "nicely behaved" Pfister extensions $K$ we 
can precisely say when $\varphi_{K}$ will be isotropic, thus providing a generalization of the case $K=F(\psi)$ with $\psi \in P_{n+1} F$ which was covered in Theorem 3.3.

Let $\Psi=\left\{\psi_{i}\right\} \subset P_{m} F$ be a non-empty set of $m$-fold Pfister forms. Following the terminology of [6], we call $\Psi$ a linked group of Pfister forms if the set $\bar{\Psi}=\left\{\psi_{i}\right.$ $\left.\left(\bmod I^{m+1} F\right)\right\}$ forms a subgroup of $I^{m} F / I^{m+1} F$. Equivalently, $\Psi$ is a linked group of Pfister forms if for any $\psi_{i}, \psi_{j} \in \Psi$ there exist $\pi_{i j} \in P_{m-1} F, x_{i}, x_{j} \in \dot{F}$, such that $\psi_{i} \simeq \pi_{i j} \otimes\left\langle\left\langle x_{i}\right\rangle\right\rangle, \psi_{j} \simeq \pi_{i j} \otimes\left\langle\left\langle x_{j}\right\rangle\right\rangle$, and $\pi_{i j} \otimes\left\langle\left\langle-x_{i} x_{j}\right\rangle\right\rangle \in \Psi$. The field $F$ is called $m$-linked if $P_{m} F$ is a linked group of Pfister forms. Let $\Psi$ be a linked group of $m$-fold Pfister forms. We say that $\left\{\psi_{i}\right\} \subset P_{m} F$ generates $\Psi$ if $\left\{\psi_{i}\left(\bmod I^{m+1} F\right)\right\}$ generates the subgroup $\bar{\Psi}$ of $I^{m} F / I^{m+1} F$. For more information on these groups, on Pfister extensions, and on linked fields in general, we refer the reader to [6] and [4].

Theorem 4.5. Let $\varphi, \pi \in W F$ be anisotropic with $\operatorname{dim} \varphi=2^{n+1}, \pi \in P_{n} F$, and $\varphi \equiv \pi\left(\bmod J_{n+1} F\right)$. Let $\left\{\psi_{i}\right\} \subset P_{n+1} F$ be a non-empty set which generates a linked group $\Psi$ of Pfister forms. Let $K=F\left(\left\{\psi_{i}\right\}\right)$. Then the following are equivalent:

(1) $\varphi_{K}$ is isotropic.

(2) There exists $\gamma \in \Psi$ such that $\varphi$ contains a Pfister neighbor of $\gamma$ (of dimension $\left.2^{n}+2^{n-1}\right)$.

Proof. Let us first assume that (2) holds. Since $\varphi$ contains a Pfister neighbor of $\gamma \in \Psi$, it is clear that $\varphi_{K}$ will be isotropic if $\gamma_{K}$ is hyperbolic. But this is true by [6, Linked Group Theorem 2.1], and (1) follows.

Now suppose that $\varphi_{K}$ is isotropic. We may assume that $\varphi$ represents 1 over $F$. Let $E=F(\pi)$ and $L=E\left(\left\{\psi_{i}\right\}\right)$. Clearly, $\varphi_{L}$ is isotropic. But $\varphi_{E} \in G P_{n+1} E$ and $\varphi_{E}$ is anisotropic by Lemma 3.1(1). In particular, $\varphi_{L} \in G P_{n+1} L$ is hyperbolic. Now $\varphi_{E}$ represents 1 , and hence $\varphi_{E} \in P_{n+1} E \cap W(L / E)$. Clearly, the set $\left\{\left(\psi_{i}\right)_{E}\right\}$ generates a linked group $\Psi_{E}$ of Pfister forms over $E$, and as sets we have $\Psi_{E}=$ $\left\{\psi_{E} \mid \psi \in \Psi\right\}$. By the Linked Group Theorem, there exists $\gamma \in \Psi$ such that $\varphi_{E} \simeq \gamma_{E}$. By Theorem 3.3, this implies that $\varphi$ contains a Pfister neighbor of $\gamma$ of dimension $2^{n}+2^{n-1}$.

(d) Some remarks on 7-dimensional forms. Now let $\varphi \in W F$ be an anisotropic 7-dimensional form, and let $c(\varphi)$ denote the Clifford invariant of $\varphi$ in the Brauer group $\operatorname{Br} F$ of $F$. Let $d=d_{ \pm} \varphi \in \dot{F} / \dot{F}^{2}$ and put $\tilde{\varphi}=\varphi \perp\langle-d\rangle$. We then have $d_{ \pm} \tilde{\varphi}=1 \in \dot{F} / \dot{F}^{2}$, i.e. $\tilde{\varphi} \in I^{2} F=J_{2} F$, and $c(\tilde{\varphi})=c(\varphi)$ (cf. [18, p.121]). It is known that $\varphi$ is a Pfister neighbor iff $c(\varphi)=1$ (see [17, p.11]). So let us from now on assume that $c(\varphi)$ has index 2, i.e. $c(\varphi)=(-x,-y)_{F} \in \operatorname{Br} F$ for some quaternion division algebra $(-x,-y)_{F}$ over $F$. Note that $c(\langle\langle x, y\rangle\rangle)=(-x,-y)_{F}$, and $\langle\langle x, y\rangle\rangle$ is anisotropic because $(-x,-y)_{F}$ is a division algebra (see [18, Ch.III, Theorem 2.7]). Also $c(\tilde{\varphi} \perp-\langle\langle x, y\rangle\rangle)=1 \in \mathrm{Br} F$, which, by Merkurjev's theorem, implies that $\tilde{\varphi} \equiv\langle\langle x, y\rangle\rangle\left(\bmod J_{3} F\right)$. Now $\varphi$ is anisotropic and $\operatorname{so} \operatorname{dim} \tilde{\varphi}_{\text {an }}=\operatorname{dim}(\varphi \perp\langle-d\rangle)_{\text {an }}$ $=6$ or 8 . Using the fact that $\tilde{\varphi}$ is a good form of height and degree 2 (which follows from $\left.\tilde{\varphi} \equiv\langle\langle x, y\rangle\rangle\left(\bmod J_{3} F\right)\right)$, or by applying Jacobson's theorem on 6 -dimensional $I^{2}$-forms (see, e.g., [20]), we see that $\tilde{\varphi}$ is in fact anisotropic. We immediately get the following consequence of our previous results.

Corollary 4.6. Let $\varphi \in W F$ be anisotropic with $\operatorname{dim} \varphi=7$ and $c(\varphi)=(-x,-y)_{F}$ in $\operatorname{Br} F$ for some quaternion division algebra $(-x,-y)_{F}$ over $F$. Let $d=d_{ \pm} \varphi$ and 
$\tilde{\varphi} \simeq \varphi \perp\langle-d\rangle$. Then $\tilde{\varphi}$ is anisotropic and $F(\varphi)$ is equivalent to $F(\tilde{\varphi})$. In particular, if $K / F$ is a field extension then $\varphi_{K}$ is isotropic iff $\tilde{\varphi}_{K}$ is isotropic.

If $\psi \in W F$ is anisotropic with $\operatorname{dim} \psi \geq 2$ and if $K=F(\psi)$, then $\varphi_{K}$ is isotropic iff $\psi$ is similar to a subform of $\tilde{\varphi}$, or $\psi$ is similar to a subform of some $\gamma \in P_{3} F$ and $\varphi$ contains a Pfister neighbor of $\gamma$.

Let $\left\{\psi_{i}\right\} \subset P_{3} F$ be a non-empty set which generates a linked group $\Psi$ of Pfister forms. Let $K=F\left(\left\{\psi_{i}\right\}\right)$. Then $\varphi_{K}$ is isotropic iff there exists $\gamma \in \Psi$ such that $\varphi$ contains a Pfister neighbor of $\gamma$.

Proof. Most of these statements follow immediately from Theorems 4.1, 4.4, 4.5. The only remaining thing to show is that if $\tilde{\varphi}$ contains a Pfister neighbor of some $\gamma \in P_{3} F$ then $\varphi$ contains a Pfister neighbhor of $\gamma$. Now if $\tilde{\varphi}$ contains a Pfister neighbor of $\gamma$ then $\tilde{\varphi}$ becomes isotropic over $F(\gamma)$ and therefore, by Theorem 4.1, $\tilde{\varphi}$ contains a 6 -dimensional Pfister neighbor $\chi$ of $\gamma$. Since $\operatorname{dim} \varphi=\operatorname{dim} \tilde{\varphi}-1$, an easy Witt index calculation shows that $\varphi$ contains a 5 -dimensional subform of $\chi$ which is, of course, also a Pfister neighbor of $\gamma$.

Let $K / F$ be a field extension. Let $\varphi \in W F$ be anisotropic. Then $\varphi$ is said to be $K$-minimal if $\varphi_{K}$ is isotropic, but for all $\eta \subset \varphi$ with $\operatorname{dim} \eta<\operatorname{dim} \varphi$ we have that $\eta_{K}$ is anisotropic. In the case $K=F(\psi)$ where $\psi \in W F$ is anisotropic with $\operatorname{dim} \psi=3, K$-minimal forms have been studied in [12]. It turns out that in this case, $K$-minimal forms always have odd dimension $\geq 3$, and that 3 -dimensional $K$-minimal forms are exactly the forms similar to $\psi$. A precise criterion for a 5 dimensional form to be $K$-minimal is also given in [12]. For 7-dimensional forms, we have the following result, which has been proved in [8]. We give a new proof here based on the results in this paper.

Proposition 4.7. Let $\varphi \in W F$ be anisotropic with $\operatorname{dim} \varphi=7$ and with $c(\varphi)=$ $(-x,-y)_{F}$ in $\operatorname{Br} F$ for some quaternion algebra $(-x,-y)_{F}$ over $F$. Let $\psi \simeq\langle 1, a, b\rangle$ $\in W F$ be anisotropic and let $K=F(\psi)$. Then $\varphi$ is not $K$-minimal, i.e. there exists $\mu \subset \varphi$ with $\operatorname{dim} \mu \leq 5$ such that $\mu_{K}$ is isotropic.

Proof. We may assume that $\varphi_{K}$ is isotropic. Note that $\psi$ is a Pfister neighbor of $\langle\langle a, b\rangle\rangle$ and that therefore $K$ is equivalent to $F(\langle\langle a, b\rangle\rangle)$. If $c(\varphi)=1$ then $\varphi$ is a Pfister neighbor of some $\gamma \in P_{3} F$. Since $\gamma_{K}$ is isotropic and hence hyperbolic, it follows that $\gamma \simeq\langle\langle a, b, r\rangle\rangle$ for some $r \in \dot{F}$. It follows easily that $\varphi$ contains a subform similar to $\langle 1, a, b\rangle$ and $\varphi$ is therefore not $K$-minimal.

So suppose now that $c(\varphi) \neq 1 \in \operatorname{Br} F$, i.e., $(-x,-y)_{F}$ is a division algebra. As above, consider $\tilde{\varphi} \simeq \varphi \perp\left\langle-d_{ \pm} \varphi\right\rangle$, which is anisotropic with $\tilde{\varphi} \equiv\langle\langle x, y\rangle\rangle$ $\left(\bmod J_{3} F\right)$, where $\langle\langle x, y\rangle\rangle$ is also anisotropic. Let $E=F(\langle\langle x, y\rangle\rangle)$. Then $\tilde{\varphi}_{E} \in G P_{3} E$ is anisotropic by Lemma 3.1(1). In particular, since $\tilde{\varphi}_{K}$ is isotropic we cannot have $\langle\langle a, b\rangle\rangle \simeq\langle\langle x, y\rangle\rangle$. Thus, $\langle\langle a, b\rangle\rangle_{E}$ is anisotropic. But clearly, $\tilde{\varphi}_{E} \in G P_{3} E$ becomes isotropic and hence hyperbolic over $E(\langle\langle a, b\rangle\rangle)$. Therefore, there exist $u, v \in \dot{E}$ such that $\tilde{\varphi}_{E} \simeq\langle\langle a, b\rangle\rangle_{E} \otimes\langle u, v\rangle$. Now $E / F$ is excellent and by [5, Proposition 2.11] we can in fact choose $u, v \in \dot{F}$. Let $\gamma \simeq\langle\langle a, b, u v\rangle\rangle \simeq u(\langle\langle a, b\rangle\rangle \otimes\langle u, v\rangle) \in P_{3} F$. Since $\gamma_{E}$ is similar to $\tilde{\varphi}_{E}$, it follows from Theorem 3.3 that $\tilde{\varphi}$ contains a Pfister neighbor of dimension 6 of $\gamma$, and as in the previous proof, $\varphi$ contains a Pfister neighbor $\mu$ of $\gamma$ of dimension 5. Now obviously $\gamma_{K}$ is hyperbolic, and thus $\mu_{K}$ is isotropic. This implies that $\varphi$ is not $K$-minimal, because $\mu \subset \varphi$ and $\operatorname{dim} \mu<\operatorname{dim} \varphi$. 
Consider two anisotropic 7-dimensional forms $\varphi_{1}$ and $\varphi_{2}$ with $c\left(\varphi_{1}\right)=c\left(\varphi_{2}\right)=$ $(-x,-y)_{F} \neq 1 \in \operatorname{Br} F$. We know by Theorem 4.4 and Corollary 4.6 that $F\left(\varphi_{1}\right)$ and $F\left(\varphi_{2}\right)$ are equivalent iff the (anisotropic) forms $\varphi_{1} \perp\left\langle-d_{ \pm} \varphi_{1}\right\rangle$ and $\varphi_{2} \perp\left\langle-d_{ \pm} \varphi_{2}\right\rangle$ are similar. We do not know, however, whether equivalence implies that $F\left(\varphi_{1}\right)$ is isomorphic to $F\left(\varphi_{2}\right)$. The following example shows that $\varphi_{1}$ need not be similar to $\varphi_{2}$. Let $F=\mathbb{R}((t))$ be the Laurent series field in one variable over the reals. Let $\tilde{\varphi} \simeq\langle 1,1,1,1,1,1, t, t\rangle$. Clearly, $\tilde{\varphi}$ is anisotropic and

$$
\tilde{\varphi} \equiv\langle\langle 1,-t\rangle\rangle \quad\left(\bmod J_{3} F\right) .
$$

Let $\varphi_{1} \simeq\langle 1,1,1,1,1,1, t\rangle$ and $\varphi_{2} \simeq\langle 1,1,1,1,1, t, t\rangle$. Then $\varphi_{1}$ and $\varphi_{2}$ are not similar, as follows readily from Springer's theorem (cf. [22, Ch.6, Corollary 2.6]).

\section{REFERENCES}

[1] J. Kr. Arason and M. Knebusch, Über die Grade quadratischer Formen, Math. Ann. 234 (1978), 167-192. MR 58:21933

[2] H. Ahmad and J. Ohm, Function fields of Pfister neighbors, J. Algebra. 178 (1995), 653-664. CMP 1996:4

[3] R. Elman and T. Y. Lam, Pfister forms and K-theory of fields, J. Algebra 23 (1972), 181213. MR 46:1882

[4] _ Quadratic forms and the u-invariant II, Invent. Math. 21 (1973), 125-137. MR 54:5114

[5] R. Elman, T. Y. Lam, and A. R. Wadsworth, Amenable fields and Pfister extensions, Proc. of Quadratic Forms Conference (ed. G. Orzech), Queen's Papers in Pure and Applied Mathematics No. 46 (1977), 445-491. MR 58:27756

[6] _ Function fields of Pfister forms, Invent. Math. 51 (1979), 61-75. MR 80m:10017

[7] R. W. Fitzgerald, Quadratic forms of height two, Trans. Amer. Math. Soc. 283 (1984), 339-351. MR 85f: 11027

[8] D. W. Hoffmann, Function Fields of Quadratic Forms, Ph.D. thesis, University of California, Berkeley, California 1992.

[9] _ Isotropy of 5-dimensional quadratic forms over the function field of a quadric, Proceedings of the 1992 Santa Barbara Summer Research Institute on Quadratic Forms and Division Algebras, Proc. Symp. Pure Math. 58.2 (1995), 217-225. CMP 1995:11

[10] _ On 6-dimensional quadratic forms isotropic over the function field of a quadric, Comm. Alg. 22 (1994), 1999-2014. MR 95g:11027

[11] _ Isotropy of quadratic forms over the function field of a quadric, Math. Z. 220 (1995), 461-476. CMP 1996:4

[12] D. W. Hoffmann, D. W. Lewis, and J. Van Geel, Minimal forms for function fields of conics, Proceedings of the 1992 Santa Barbara Summer Research Institute on Quadratic Forms and Division Algebras, Proc. Symp. Pure Math. 58.2 (1995), 227-237. CMP 1995:11

[13] J. Hurrelbrink and U. Rehmann, Splitting patterns of quadratic forms, Math. Nachr. 176 (1995), 111-127. CMP 1996:4

[14] O.T. Izhboldin, On the nonexcellence of field extensions $F(\pi) / F$, preprint (1995).

[15] B. Kahn, Formes quadratiques de hauteur et de degré 2, to appear in: Indag. Math.

[16] M. Knebusch, Generic splitting of quadratic forms I, Proc. London Math. Soc. 33 (1976), 65-93. MR 54:230

[17] , Generic splitting of quadratic forms II, Proc. London Math. Soc. 34 (1977), 1-31. MR 55:379

[18] T. Y. Lam, The Algebraic Theory of Quadratic Forms, Reading, Massachusetts: Benjamin 1973 (revised printing 1980). MR 53:277; MR 83d:10022

[19] D. Leep, Function field results, handwritten notes taken by T. Y. Lam (1989).

[20] P. Mammone and D. B. Shapiro, The Albert quadratic form for an algebra of degree four, Proc. Amer. Math. Soc. 105 (1989), 525-530. MR 89g:16029

[21] J. Ohm, The Zariski problem for function fields of quadratic forms, to appear in: Proc. Amer. Math. Soc. 
[22] W. Scharlau, Quadratic and Hermitian Forms, Berlin, Heidelberg, New York, Tokyo: Springer 1985. MR 86k:11022

[23] A. R. Wadsworth, Similarity of quadratic forms and isomorphism of their function fields, Trans. Amer. Math. Soc. 208 (1975), 352-358. MR 51:12702

Aindorferstr. 84, D-80689 Munich, Germany

Current address: Laboratoire de Mathématiques, Faculté des Sciences, Université de FrancheComté, 25030 Besançon Cedex, France

E-mail address: detlev@math.univ-fcomte.fr 